\title{
Universiteit
}

Leiden

The Netherlands

\section{Statistics of fluctuating colloidal fluid-fluid interfaces}

Villeneuve, V.W.A. de; Leeuwen, J.M.J. van; Saarloos, W. van

\section{Citation}

Villeneuve, V. W. A. de, Leeuwen, J. M. J. van, \& Saarloos, W. van. (2008). Statistics of fluctuating colloidal fluid-fluid interfaces. Journal of Chemical Physics, 129(16), 164710. doi:10.1063/1.3000639

Version: $\quad$ Not Applicable (or Unknown)

License: $\quad$ Leiden University Non-exclusive license

Downloaded from: https://hdl.handle.net/1887/66529

Note: To cite this publication please use the final published version (if applicable). 


\section{Statistics of fluctuating colloidal fluid-fluid interfaces}

V. W. A. de Villeneuve, J. M. J. van Leeuwen, W. van Saarloos, and H. N. W. Lekkerkerker

Citation: The Journal of Chemical Physics 129, 164710 (2008); doi: 10.1063/1.3000639

View online: https://doi.org/10.1063/1.3000639

View Table of Contents: http://aip.scitation.org/toc/jcp/129/16

Published by the American Institute of Physics

\section{Articles you may be interested in}

Dynamics of thermally driven capillary waves for two-dimensional droplets

The Journal of Chemical Physics 132, 174701 (2010); 10.1063/1.3374437

Correlation functions in the capillary wave model of the liquid-vapor interface

The Journal of Chemical Physics 82, 972 (1985); 10.1063/1.448474

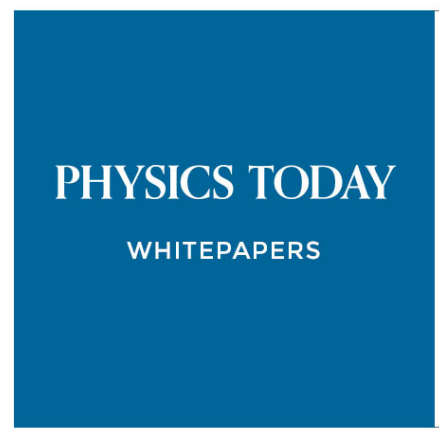




\title{
Statistics of fluctuating colloidal fluid-fluid interfaces
}

\author{
V. W. A. de Villeneuve, ${ }^{1, a)}$ J. M. J. van Leeuwen, ${ }^{2}$ W. van Saarloos, ${ }^{2}$ and \\ H. N. W. Lekkerkerker ${ }^{1}$ \\ ${ }^{1}$ Van 't Hoff Laboratory for Physical and Colloid Chemistry, University of Utrecht, Padualaan 8 , \\ 3584 CH Utrecht, The Netherlands \\ ${ }^{2}$ Instituut-Lorentz for Theoretical Physics, Leiden University, Niels Bohrweg 2, Leiden 2333 CA, \\ The Netherlands
}

(Received 17 June 2008; accepted 23 September 2008; published online 29 October 2008)

\begin{abstract}
Fluctuations of the interface between coexisting colloidal fluid phases have been measured with confocal microscopy. Due to a very low surface tension, the thermal motions of the interface are so slow that a record can be made of the positions of the interface. The theory of the interfacial height fluctuations is developed. For a host of correlation functions, the experimental data are compared with the theoretical expressions. The agreement between theory and experiment is remarkably good. (C) 2008 American Institute of Physics. [DOI: 10.1063/1.3000639]
\end{abstract}

\section{INTRODUCTION}

The study of interfaces has a long and interesting history. In 1894 van der Waals ${ }^{1}$ proposed an interface theory, which leads to a flat interface with a density profile in the direction of gravity. This result is sometimes referred to as the intrinsic interface. von Schmoluchowski ${ }^{2}$ realized that the thermal motion of the molecules induces height fluctuations in the interface. These motions have been called capillary waves, since they derive from an interplay of gravity and surface tension, such as capillary rise. The fluctuations were first treated theoretically and experimentally by Mandelstam. ${ }^{3} \mathrm{He}$ pointed out that the interface width diverges due to the shortwavelength capillary waves. This fact was rediscovered by Buff et al. ${ }^{4} 50$ years later, after which it obtained a prominent place in the discussion of interfaces. Weeks ${ }^{5}$ later pointed out that the notion of capillary waves only applies to wavelengths larger than the fluid correlation length, which is of the order of the interparticle distance.

The experimental study of interfaces was undertaken by $\operatorname{Raman}^{6}$ and Vrij ${ }^{7}$ with light scattering and starting with Braslau and co-workers ${ }^{8-10}$ by $x$-ray scattering. Although scattering on interfaces is most valuable, it always yields global information on the fluctuations, while a photographic inspection gives local information. However, the wavelengths and heights involved in the capillary waves of molecular fluids are way out of the reach of detection by photographic methods. The visual inspection of capillary waves initially remained restricted to computer simulations of interfaces in molecular systems. ${ }^{11}$

The field obtained another dimension by recent experiments of Aarts and co-workers ${ }^{12-15}$ in which they obtained pictures of fluctuating colloidal interfaces. The key is that, by lowering the surface tension to the $\mathrm{nN} / \mathrm{m}$ range, the characteristic length and time scale of the fluctuations become accessible by confocal microscopy. This opened up the possibility to follow locally the motion of the height of the

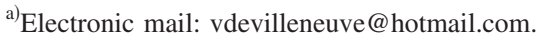

interface and to do a statistical analysis of its temporal and spatial behaviors. Of course the method has its inherent restrictions. Just as in ordinary movie recording, the pixels have a finite distance and the snapshots have to be taken at finite time intervals. For colloidal interfaces this interval can be made much smaller than the intrinsic time scale of the motions. Thus the Brownian character of the motion could be demonstrated ad oculos.

Fluctuating interfaces present also the interesting problem of determining the so-called persistence exponent, ${ }^{16}$ which characterizes the long-time power law decay of the probability that a fluctuating interface stays above a certain height. The determination of this probability amounts to calculating the probability of first crossing that height. Even though the long-time persistence exponent is a well-studied problem about which much is known, ${ }^{16,17}$ the determination of the first passage probability of a general Gaussian process with known autocorrelation function was deemed a classic unsolved problem in probability theory. ${ }^{16,17}$ In this paper we do not focus on the value of the persistence exponent at long times but rather on the first passage distribution over time scales, which can be probed in detail in the experiments that we present. We discuss the measurement of the first passage distribution and we give a partial solution to its calculation from the autocorrelation function.

In the confocal microscopy a two-dimensional section is inspected perpendicular to the interface and the density profile between the two phases is observed. A schematic of the experiment is shown in Fig. 1(a). A very precise location of the interface can be obtained by fitting the intensity with a van der Waals-like profile: $I(z, x)=a+b \tanh ([z-h(x)] / c)$, where $z$ is the direction perpendicular to the interface and $x$ is a coordinate along the interface. In the upper phase the density approaches a value corresponding to $a+b$ and in the lower phase to $a-b$, while $c$ measures the intrinsic width of the interface. Thus at every snapshot a function $h(x)$ follows and the sequence of snapshots gives the function $h(x, t)$. This is a practical separation of the particle motions, which lead at short scales to the intrinsic interface and the particle mo- 


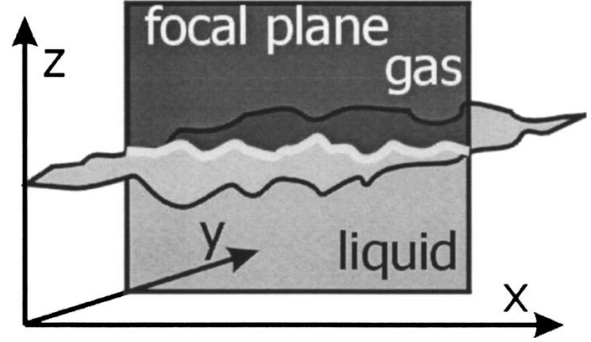

FIG. 1. Schematic view of confocal microscopy. The confocal microscope thin focal planes of approximately $500 \mathrm{~nm}$ thickness can be imaged. This enables the investigation of local phenomena such as height fluctuations

tions, which drive the long wavelength capillary waves. One might think that, if the time interval of the snapshots is sufficiently small with respect to the characteristic time scale of variation in $h(x, t)$, one can analyze $h(x, t)$ as a continuous function of the time, like a movie gives the impression of continuous motions, while it is a succession of snapshots. In a previous short report ${ }^{18}$ on these experiments we have pointed out that the statistics remains dependent on the time interval, due to the Brownian character of the motion.

We have performed confocal microscopy measurements on phase separated colloid-polymer mixtures. The colloids are $69 \mathrm{~nm}$ radius fluorescently labeled polymethyl metacrylate particles, suspended in cis/trans decalin, with polystyrene (estimated radius of gyration $=42 \mathrm{~nm})^{19}$ added as a depletant polymer. Due to a depletion induced attraction these mixtures phase separate at sufficiently high colloid and polymer volume fractions and a proper colloid to polymer aspect ratio into a colloid-rich/polymer-poor (colloidal liquid) and a colloid-poor/polymer-rich (colloidal gas) phase. ${ }^{20}$ Here the polymer concentration acts as an inverse temperature. By diluting several phase separating samples with its solvent decalin, the phase diagram was constructed. With a Nikon E400 microscope equipped with a Nikon C1 confocal scanhead, a series of 10000 snapshots of the interface was recorded at constant intervals $\Delta t$ of 0.45 and $0.50 \mathrm{~s}$ of two statepoints, which we denote as II and IV, following the notation in an earlier publication, ${ }^{18}$ to which we also refer for a discussion of the full phase diagram. The pixels are separated by a distance $\Delta x=156 \mathrm{~nm}$ and a single scan takes approximately $0.25 \mathrm{~s}$ to complete.

The setup of the paper is as follows. We start out by discussing the spatial behavior of the data of a single time frame, which requires only equilibrium statistics. The correlation functions and the statistics of hills and valleys in the interface are determined and compared to the theory.

Then we identify the set of interface modes via the Fourier decomposition

$$
h(\mathbf{x}, t)=\sum_{\mathbf{k}} h_{\mathbf{k}}(t) \exp (i \mathbf{k} \cdot x) .
$$

The modes are overdamped in the relevant regime and follow from the macroscopic interface dynamics. The motion obeys not only the macroscopic equations but is also influenced by noise. We introduce thermal noise through the Langevin equation and calculate the essential height-height correlation function $\langle h(\mathbf{0}, 0) h(\mathbf{x}, t)\rangle$. Via the equivalent
Fokker-Planck equation the probabilities on sequences ("histories") of snapshots are determined. The analysis of the distributions of "hills" and "valleys" in the time domain with respect to a level $h$ is similar to the spatial behavior. A special concern is the dependence of the residence time and the waiting time on the used time interval.

The paper closes with a discussion of the main results.

\section{EQUAL TIME CORRELATIONS}

The function $h(\mathbf{x}, t)$ provides a mathematical division between the two coexisting phases, which form the interface. The interface is of the solid-on-solid type since so-called overhangs, well known in lattice theory, are excluded by construction, as to every value of the horizontal coordinate $\mathbf{x}$ and time $t$ one unique height $h(\mathbf{x}, t)$ is associated. The basic function is the height-height correlation function. Due to translational invariance the modes $\mathbf{k}$ are independent and thus the correlation function in space has the Fourier decomposition

$$
\langle h(0,0) h(\mathbf{x}, 0)\rangle=\sum_{\mathbf{k}}\left\langle\left|h_{\mathbf{k}}^{2}\right|\right\rangle \exp (i \mathbf{k} \cdot x)
$$

The brackets denote equilibrium averages and $h_{\mathbf{k}}$ is the amplitude of the $k$ th mode. The distribution of the $h_{\mathbf{k}}$ follows from the Boltzmann factor involving the energy of a deformation of the interface, which is given by the drumhead model

$$
\mathcal{H}(\{h\})=\frac{1}{2} \int d \mathbf{x}\left[\Delta \rho g h^{2}(\mathbf{x})+\gamma(\nabla h(\mathbf{x}))^{2}\right] .
$$

Here $\Delta \rho$ is the density difference between the coexisting phases, $\gamma$ is the surface tension, and $g$ is the gravitational acceleration. The first term gives the gravitational potential energy and the second term gives the extra interfacial energy due to increase in the surface. Expressed in terms of the amplitudes $h_{\mathbf{k}}$,

$$
\mathcal{H}(\{h\})=\frac{L^{2}}{2} \sum_{\mathbf{k}}\left[\Delta \rho g+\gamma k^{2}\right]\left|h_{\mathbf{k}}\right|^{2},
$$

where $L^{2}$ is the area of the interface. ${ }^{21}$ Since Eq. (4) is quadratic in the amplitudes $h_{\mathbf{k}}$, it implies a Gaussian distribution for the $h_{\mathbf{k}}$ as follows:

$$
P_{e}\left(h_{\mathbf{k}}\right)=\frac{\exp -\left|h_{\mathbf{k}}\right|^{2} / 2\left\langle\left|h_{\mathbf{k}}\right|^{2}\right\rangle}{\left[2 \pi\left\langle\left|h_{\mathbf{k}}\right|^{2}\right\rangle\right]^{1 / 2}},
$$

with the average

$$
\left\langle\left|h_{\mathbf{k}}\right|^{2}\right\rangle=\frac{k_{B} T}{L^{2}\left(\Delta \rho g+\gamma k^{2}\right)} .
$$

With the distribution (5) of the $h_{\mathbf{k}}$, we can calculate the distribution of the heights $h$, which becomes also a Gaussian

$$
P_{\mathrm{eq}}(h)=\frac{\exp \left(-h^{2} / 2\left\langle h^{2}\right\rangle\right)}{\left[2 \pi\left\langle h^{2}\right\rangle\right]^{1 / 2}},
$$

with the mean square height $\left\langle h^{2}\right\rangle$ given by 


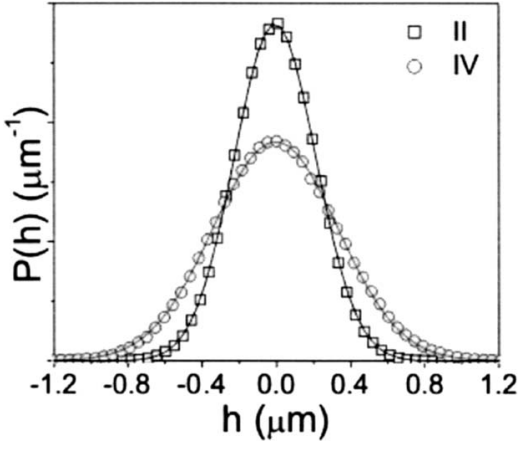

FIG. 2. The height distribution for statepoints II and IV as found experimentally. The lines are Gaussian fits to the data.

$$
\left\langle h^{2}\right\rangle=\sum_{\mathbf{k}}\left\langle h_{\mathbf{k}}^{2}\right\rangle=\frac{k_{B} T}{4 \pi \gamma} \ln \frac{1+k_{\max }^{2} \xi^{2}}{1+k_{\min }^{2} \xi^{2}} .
$$

$\xi$ is the capillary length defined as

$$
\xi^{2}=\frac{\gamma}{g \Delta \rho} .
$$

The integral has been given an upper bound $k_{\max } \simeq 2 \pi / d$ with $d$ the diameter of the particles and a lower bound $k_{\min }$ $\simeq 2 \pi / L$ due to the finite size of the interface. The lower bound can be set equal to 0 for all practical purposes, but the upper bound is essential for the convergence of the integral. Cutting off the capillary waves at the short-wavelength side is the poor man's way to handle the otherwise diverging interface width $\left\langle h^{2}\right\rangle$. There are two options to determine $\left\langle h^{2}\right\rangle$. The first follows from a fit to $P_{\text {eq }}(h)$, which is shown in Fig. 2 . The second is a direct evaluation of $\left\langle h^{2}\right\rangle$ from the recorded data. The latter always gives a $1 \%-3 \%$ larger value, which we attribute to optical artifacts due to confocal slicing. So we are inclined to prefer the former value, which amounts to $\left\langle h^{2}\right\rangle=0.219$ for statepoint II and $0.336(\mu \mathrm{m})^{2}$ for statepoint IV. Then Eq. (8) can be used to estimate the upper cutoff. On the basis of a determination of $\gamma$ (see below) one finds values around $\kappa=k_{\max } \xi \simeq 45$, but this value is rather sensitive to small variations in $\gamma$ : A variation of $\gamma$ by $10 \%-15 \%$ results in a shift in $\kappa$ by a factor 2 .

The correlation function $\langle h(\mathbf{0}, 0) h(\mathbf{x}, 0)\rangle$ is of course also measurable. In the Appendix we discuss the integral (2); here we give the result with the cutoff sent to $\infty$ as follows:

$$
\langle h(\mathbf{0}, 0) h(\mathbf{x}, 0)\rangle=\frac{k_{B} T}{2 \pi \gamma} K_{0}(x / \xi) .
$$

The divergence for $x \rightarrow 0$ of the modified Bessel function $K_{0}$ corresponds to the divergence of the interface width without a cutoff. A fit of the correlation function to the Bessel function (with a slight modification due to the cutoff) is shown in Fig. 3. Apart from a few initial points the function fits quite well. We find fitting parameters $\xi=8.0 \mu \mathrm{m}$ for statepoint II and $\xi=6.1 \mu \mathrm{m}$ for statepoint IV. The values for $\gamma$ turn out to be $58 \mathrm{nN} / \mathrm{m}$ for statepoint II and $21 \mathrm{nN} / \mathrm{m}$ for statepoint IV.

Fourier transforming the correlation function back to the wavenumber domain should lead to expression (6) as a function of $k$. However, an inverse Fourier transform requires accurate data for a large domain and the correlation function

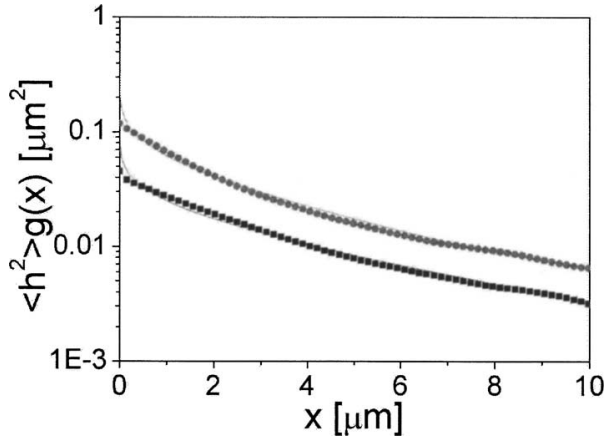

FIG. 3. The spatial correlation function $\langle h(x, 0) h(0,0)\rangle$ for the statepoints II (upper points) and IV (lower points) fitted to expression (10) using a cutoff.

is unreliable for large distances (not shown in Fig. 3). This prevents a direct check of the drumhead Hamiltonian.

\section{MULTIPLE CORRELATION FUNCTIONS}

Persistence times require the determination of multiple correlations functions. As the data are stored for all sampled positions we can determine the probability density on a sequence of events as follows:

$G_{n}\left(h_{1}, \mathbf{x}_{1} ; \ldots ; h_{n}, \mathbf{x}_{n}\right)=\left\langle\delta\left(h\left(\mathbf{x}_{1}, 0\right)-h_{1}\right) \cdots \delta\left(h\left(\mathbf{x}_{n}, 0\right)-h_{n}\right)\right\rangle$,

which gives the joint probability that the interface at position $\mathbf{x}_{1}$ has the height $h_{1}$ and subsequently at position $\mathbf{x}_{i}$ the height $h_{i}$, etc. A straightforward evaluation of Eq. (11) proceeds via writing the $\delta$ functions as a Fourier integral and then expressing $h(\mathbf{x}, 0)$ in terms of the amplitudes $h_{\mathbf{k}}$. As all integrals are over a quadratic form in the exponent the result is a Gaussian in the $h_{i}$. It leads to the expression

$$
\begin{aligned}
& G_{n}\left(h_{1}, \mathbf{x}_{1} ; \ldots ; h_{n}, \mathbf{x}_{n}\right)=\left(\frac{\operatorname{det} g^{-1}}{\left(2 \pi\left\langle h^{2}\right\rangle\right)^{n}}\right)^{1 / 2} \\
& \times \exp \left[-\frac{1}{2} \sum_{i, j} g_{i, j}^{-1} \frac{h_{i} h_{j}}{\left\langle h^{2}\right\rangle}\right] .
\end{aligned}
$$

In this notation the matrix $g_{i, j}$ is the correlation matrix

$$
g_{i, j}=g\left(\left|\mathbf{x}_{i}-\mathbf{x}_{j}\right|, 0\right),
$$

which turns out to be the equal-time value of the heightheight correlation function

$$
g\left(\left|\mathbf{x}-\mathbf{x}^{\prime}\right|, t-t^{\prime}\right)=\left\langle h(\mathbf{x}, t) h\left(\mathbf{x}^{\prime}, t^{\prime}\right)\right\rangle /\left\langle h^{2}\right\rangle .
$$

A shortcut for deriving result (12) is to evaluate the following integral in two ways:

$$
\int d h_{1} \cdots d h_{n} h_{i} h_{j} G\left(h_{1}, \mathbf{x}_{1} ; \ldots ; h_{n}, \mathbf{x}_{n}\right)=\left\langle h^{2}\right\rangle g_{i, j} .
$$

The first uses definition (11) and obviously leads to the right hand side of Eq. (15). The second way uses expression (12). Then one has to diagonalize the quadratic form in the exponent and the integration over the eigendirections also leads to the right hand side of Eq. (15), which shows that Eq. (13) is correct.

In Eq. (14) and accordingly in result (12), we have factored out $\left\langle h^{2}\right\rangle$ because we want to use it as a scale for the 
heights. Equation (12) shows that the correlation function $g$ dictates the behavior of the multiple correlation functions.

To give an impression on the behavior of the $G$ 's we first consider a few small values of $n$. For a single position $(n$ $=1$ ) the value $g(0,0)=1$ and Eq. (12) reduces to the equilibrium height distribution (5) as follows:

$$
G_{1}\left(h_{1}\right)=P_{\text {eq }}\left(h_{1}\right),
$$

which is shown in Fig. 2. From this probability we derive the important probabilities $q^{+}(h)$ to find a height above $h$ and $q^{-}(h)$ for finding a height below $h$. They are given by the expressions

$$
q^{+}(h)=\int_{h}^{\infty} P_{\mathrm{eq}}\left(h_{1}\right) d h_{1}, \quad q^{-}(h)=\int_{-\infty}^{h} P_{\mathrm{eq}}\left(h_{1}\right) d h_{1} .
$$

In integrals such as Eq. (17), one changes of course to the combination $h_{1} /\left\langle h^{2}\right\rangle^{1 / 2}$ as an integration variable, such that the $q^{ \pm}(h)$ become functions of the scaled variable $h /\left\langle h^{2}\right\rangle^{1 / 2}$. The result of the integration in Eq. (17) is an error function in this parameter. From now on we work with these reduced heights.

The probability density $G$ for $n=2$ is still sufficiently simple to make it explicit. The matrix $g_{i, j}$ and its inverse $g_{i, j}^{-1}$ read

$$
g_{i, j}=\left(\begin{array}{cc}
1 & g_{1,2} \\
g_{2,1} & 1
\end{array}\right)
$$

and

$$
g_{i, j}^{-1}=\frac{1}{1-g_{1,2}^{2}}\left(\begin{array}{cc}
1 & -g_{1,2} \\
-g_{2,1} & 1
\end{array}\right) .
$$

So $G_{2}$ follows from the general definition as

$$
G_{2}\left(h_{1}, \mathbf{0} ; h_{2}, \mathbf{x}\right)=\frac{1}{2 \pi\left[1-g^{2}\right]^{1 / 2}} \exp \left[-\frac{h_{1}^{2}-2 g h_{1} h_{2}+h_{2}^{2}}{2\left[1-g^{2}\right]}\right],
$$

with $g$ a shorthand for $g_{1,2}=g(x, 0)$ and $x$ the distance of sampling. Note that this expression is symmetric in the entries $h_{1}$ and $h_{2}$ and that dependence only enters through $g$ $=g(x, 0)$.

\section{STATISTICS OF SEQUENCES}

The probability densities (11) are measurable, but the statistics becomes poor when too much entries are taken. Therefore integrated probabilities are more accessible. For what follows it is interesting to study the probability that a sequence in space of precisely $n$ successive values occurs of the heights above the level $h$. The above given theory implies that it is given by the ratio of two integrals

$$
p_{n}^{+}(h)=q^{-(n+)-}(h) / q^{+-}(h),
$$

which will be shown below. In this notation the superscript prescribes the integration domain. The numerator of Eq. (21) reads

$$
\begin{aligned}
q^{-(n+)-}(h)= & \int_{-\infty}^{h} d h_{0} \int_{h}^{\infty} d h_{1} \ldots \int_{h}^{\infty} d h_{n} \\
& \times \int_{-\infty}^{h} d h_{n+1} G_{n+2}\left(h_{0}, \ldots, h_{n+1}\right) .
\end{aligned}
$$

The integral over the first variable $h_{0}$ guarantees that the sequence starts below level $h$, the next $n$ integrations select points above the level $h$, and the sequence ends with $h_{n+1}$ below level $h$. So the numerator in Eq. (21) selects the hills of precisely $n$ consecutive values of the height above level $h$. We have omitted in $G_{n+2}$ the position arguments since it is understood that points are equidistant. So the sequence of values $g(m \Delta x, 0)$ enters, with $0 \leq m<n+2$. The denominator is the integral

$$
q^{+-}(h)=\int_{h}^{\infty} d h_{1} \int_{-\infty}^{h} d h_{2} G_{2}\left(h_{1}, h_{2}\right) .
$$

It counts the number of hills since each hill is followed by a transition from above to below the level $h$. The denominator serves as a normalizing factor. Summing Eq. (21) over $n$ (from 1 to $\infty$ ) gives the total number of hills above $h$ and as this equals the number of crossings, we see that distribution (21) is normalized. Thus expression (21) is the normalized probability distribution for $n$ successive points above the level $h$.

The average length $\chi^{+}(h)$ of a sequence is defined as

$$
\chi^{+}(h)=\sum_{n=1} n p_{n}^{+}(h) .
$$

We also looked to sequences below the height $h$. They are given by the probability $p_{n}^{-}(h)$, which follows from a similar definition as Eq. (21), with + and - interchanged. The updown symmetry of the problem yields the relation

$$
p_{n}^{-}(h)=p_{n}^{+}(-h) \text {. }
$$

The average length $\chi^{-}(h)$ of a stretch below $h$ similarly equals

$$
\chi^{-}(h)=\sum_{n=1} n p_{n}^{-}(h)
$$

Inserting expression (21) into definition (24) for $\chi^{+}(h)$, the numerator in Eq. (22) is multiplied by the number of values larger than $h$. Summation over all $n$ leads to the average number of points above the level $h$, which is given by integral (17). Thus we arrive at the relations

$$
\chi^{ \pm}(h)=q^{ \pm}(h) / q^{+-}(h) .
$$

The remarkable point about these relations is that, although the probabilities $p_{n}^{ \pm}(h)$ are given by multiple integrals, the averages $\chi^{ \pm}(h)$ result from simple integrals. The $q^{ \pm}(h)$ are error functions and $q^{+-}$is a twofold integral involving the function $G_{2}$, thus containing only the value $g(\Delta x, 0)$.

A trivial result from Eq. (27) is that the ratio $\chi^{+}(h) / \chi^{-}(h)$ is the same as the ratio $q^{+}(h) / q^{-}(h)$. Both give the ratio of 


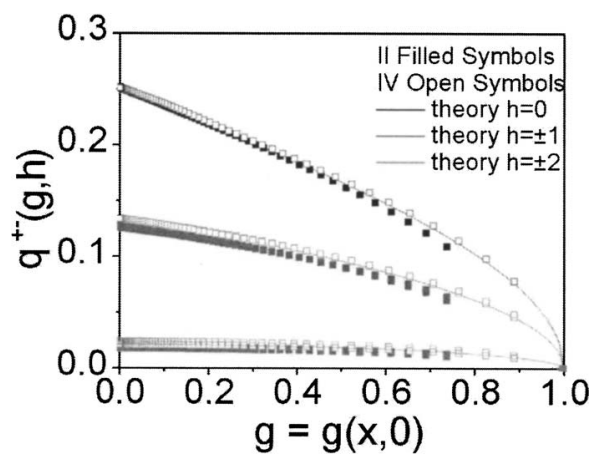

FIG. 4. The function $q^{+-}(h, g)$ as a function of $x$ through $g=g(x, 0)$, for some values of $h$ (in units $\left\langle h^{2}\right\rangle^{1 / 2}$ ). The drawn lines are the calculated values and the points are the measured values.

the total number of points above and below the level $h$. As the $q^{+}(h)$ and $q^{-}(h)$ add up to 1 , a more intriguing result follows for the sum

$$
\chi^{+}(h)+\chi^{-}(h)=\frac{1}{q^{+-}(h, g)} .
$$

Deliberately we have given $q^{+-}$also the argument $g$, which incorporates the spatial dependence on $x$. Tacitly we have assumed that this distance is the sampling distance $\Delta x$. But nothing prevents us from taking a multiple $n$ of $\Delta x$. Then $g$ will refer to $g(n \Delta x, 0)$. In Fig. 4 we have plotted the experimental values of $q^{+-}(h, g)$ for various values of $g(n \Delta x, 0)$, which we take as a parameter on the horizontal axis. The curves are the calculated values of $q^{+-}(h, g)$. We have not found a closed expression for $q^{+-}$in terms of known functions, but a number of limits are explicitly obtainable. The $g$ dependence is exemplified by the case $h=0$, which reads

$$
q^{+-}(0, g)=\frac{1}{2}-\frac{1}{\pi} \arctan \left(\frac{1+g}{1-g}\right)^{1 / 2} .
$$

The $h$ dependence is by and large controlled by the limiting behavior

$$
\begin{aligned}
& q^{+-}(h, 0)=q^{+}(h) q^{-}(h), \\
& q^{+-}(h, g \rightarrow 1) \simeq \frac{\sqrt{1-g}}{\pi \sqrt{2}} \exp \left[-\frac{h^{2}}{2\left\langle h^{2}\right\rangle}\right] .
\end{aligned}
$$

Apart from the averages also the individual $p_{n}^{ \pm}(h)$ can be measured and compared with theoretical expressions (21). In Fig. 5 we show $p_{n}^{+}(h)$ for statepoint IV as a function of $h$ for a number of $n$. The theory requires the evaluation of multiple

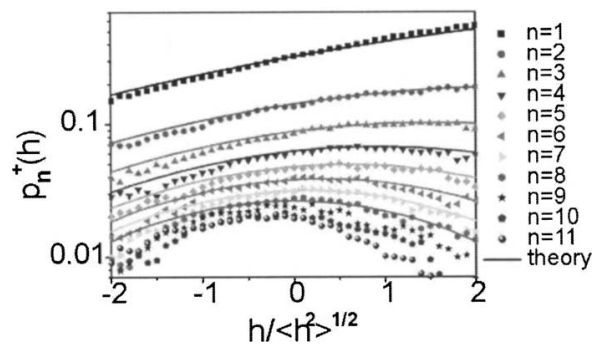

FIG. 5. The spatial $p_{n}^{+}(h)$ as a function of $h$ for a number of $n$ for statepoint IV. The drawn lines are the theoretical values. integrals (22), which can be carried out by Monte Carlo integration. The best procedure is to generate a distribution according to the Gaussian integrand and then reject the points that fall outside the integration domain. This technique also applies to correlation functions for other histories with another integration domain.

The theoretical curves are calculated up to $n=8$, which is a practical limit. By the Monte Carlo integration we could add a few more values, but the asymptotic behavior for large $n$ falls of course outside the range of this method. The agreement between theory and experiment is good, but typically there are deviations for larger negative values of $h$, where the experimental points are systematically lower than the theoretical prediction.

\section{THE DYNAMIC INTERFACE MODES}

As Secs. III and IV show, the interface fluctuations have a rich spatial structure. So it is an interesting question how this compares with the interface fluctuations in time. The temporal development of the interface is determined by the macroscopic equations for the interface modes as well as by the influence of thermal noise. In this section we briefly discuss the interface modes and in Sec. VI we treat the noise.

The problem of the interface modes has been addressed by Jeng et al., ${ }^{22}$ who have made an extensive study of the interface modes in the various regimes distinguished by the relative strength of viscosity and surface tension. The modes are overdamped for our experimental conditions and decay as

$$
h_{\mathbf{k}}(t)=h_{\mathbf{k}} \exp \left(-\omega_{k} t\right),
$$

with a rate

$$
\omega_{k}=\frac{1}{2 t_{c}}\left[(k \xi)^{-1}+k \xi\right]
$$

where the capillary time $t_{c}$ is given by

$$
t_{c}=\frac{\left(\eta+\eta^{\prime}\right)}{\sqrt{g \gamma \Delta \rho}} .
$$

Here $\eta$ and $\eta^{\prime}$ are the viscosities of the lower and upper fluids. A few remarks on Eq. (31) are worth making.

- The dispersion relation $\omega_{k}$ is in general rather complicated. Simplification (32) derives from the approximation $\rho \omega_{k} \ll \eta k^{2}$, which is very well fulfilled for colloidal interfaces with extremely low surface tension. The approximation is controlled by the number

$\frac{\xi}{L_{\eta}}=\left(\frac{\gamma^{3} \Delta \rho}{g}\right)^{1 / 2} \frac{1}{\left(\eta+\eta^{\prime}\right)^{2}}$,

which is the ratio of the capillary length $\xi$ to the viscous length $L_{\eta}=\left(\eta+\eta^{\prime}\right)^{2} / \gamma \Delta \rho$. It is very small, $10^{-5}$, for colloidal interfaces, while it is very large for, e.g., water $\left(10^{5}\right)$.

- The spectrum has a slowest mode with wavelength $\xi$ and decay rate $t_{c}$, in contrast to the capillary waves of molecular fluids, where the modes become slower the 
longer the wavelength. This mode starts to dominate the behavior of the correlations for long times.

\section{THE LANGEVIN EQUATION}

The thermal influences can be incorporated by a fluctuating force $F_{\mathbf{k}}(t)$ on mode $\mathbf{k}$ in the Langevin equation ${ }^{23}$

$$
\frac{\partial h_{\mathbf{k}}}{\partial t}=-\omega_{k} h_{\mathbf{k}}+F_{\mathbf{k}}(t)
$$

The first term on the right hand side is the systematic damping force, which by itself would lead to an exponential decay of mode $k$. The random force $F_{\mathbf{k}}(t)$ has a zero average and is assumed to be $\delta$ correlated in time (white noise),

$$
\left\langle F_{\mathbf{k}}(t) F_{\mathbf{k}^{\prime}}\left(t^{\prime}\right)\right\rangle=\delta_{\mathbf{k}+k^{\prime}, 0} \Gamma_{\mathbf{k}} \delta\left(t-t^{\prime}\right),
$$

where $\Gamma_{\mathbf{k}}$ can be found from the fluctuation-dissipation theorem

$$
\frac{\Gamma_{\mathbf{k}}}{2 \omega_{k}}=\left\langle\left|h_{\mathbf{k}}\right|^{2}\right\rangle
$$

Langevin equation (35) assumes that the slow capillary waves form a complete set to characterize the motion of the interface. $\Gamma_{\mathbf{k}}$ is linked in Eq. (37) to the equilibrium average of the amplitudes $h_{\mathbf{k}}$, which is given by Eq. (5).

With the Langevin equation all time-dependent correlation functions can be calculated. In particular, the heightheight correlation function follows as

$$
\langle h(\mathbf{0}, 0) h(\mathbf{x}, t)\rangle=\left\langle h^{2}\right\rangle g(x, t)=\sum_{\mathbf{k}}\left\langle\left|h_{\mathbf{k}}\right|^{2}\right\rangle \exp \left(i \mathbf{k} \cdot \mathbf{x}-\omega_{k} t\right),
$$

with $\left\langle\left|h_{\mathbf{k}}\right|^{2}\right\rangle$ given by Eq. (6) and $\left\langle h^{2}\right\rangle$ by Eq. (8). Note that for this correlation function the influence of the fluctuating force $F_{\mathbf{k}}(t)$ averages out such that it depends only on the macroscopic dynamics of the interface. It involves, apart from the decay rate $\omega_{k}$, only the thermal average $\left\langle\left|h_{\mathbf{k}}\right|^{2}\right\rangle$. Some properties of the integral yielding this function are listed in the Appendix.

The first point is the determination of $\gamma$ and $t_{c}$ from the data. We represent $\langle h(\mathbf{0}, 0) h(\mathbf{0}, t)\rangle$ as

$$
\langle h(\mathbf{0}, 0) h(\mathbf{0}, t)\rangle=\frac{k_{B} T}{2 \pi \gamma} H\left(t / t_{c}, \kappa\right) .
$$

Here again $\kappa=k_{\max } \xi$. In the Appendix we prove that

$$
H\left(t / t_{c}, \infty\right)=K_{0}\left(t / t_{c}\right),
$$

with $K_{0}$ the modified Bessel function of order 0. For $t$ $\geq t_{c} / \kappa$, the function $H\left(t / t_{c}, \kappa\right)$ is well represented by $K_{0}\left(t / t_{c}\right)$. Since $\kappa$ is of the order 40-50 (see Sec. II), expression (40) suffices for most of the measured points, except of course for the first few points near $t=0$, where the right hand side of Eq. (40) diverges. Leaving them out for the moment, we find from a fit for statepoint II: $t_{c}=20 \mathrm{~s}$ and $\gamma$ $=66 \mathrm{nN} / \mathrm{m}$ and for statepoint IV: $t_{c}=33 \mathrm{~s}$ and $\gamma$ $=22 \mathrm{nN} / \mathrm{m}$, which compare well with the values found from the spatial dependence. Effectively $t_{c}$ acts as a horizontal scale parameter and $\gamma$ as a vertical shift. $t_{c}$ is mainly deter-

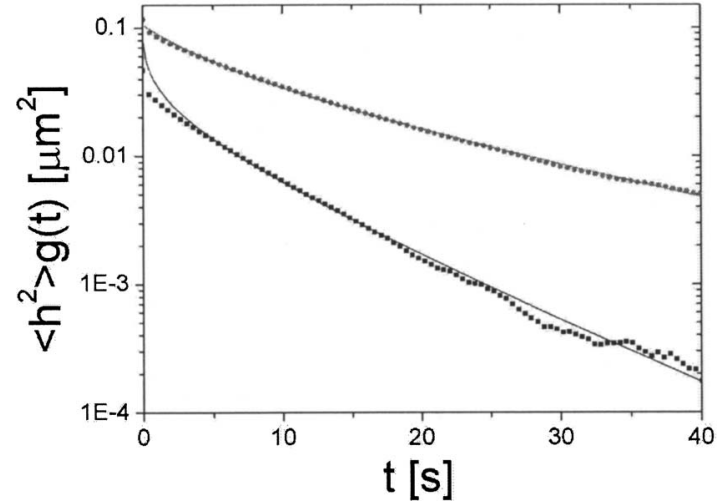

FIG. 6. The correlation function $\langle h(0, t) h(0,0)\rangle$. The points are the experimental values and the lines are the curves according to Eq. (40) using a cutoff.

mined by the asymptotic behavior, while $\gamma$ is more sensitive to the initial behavior.

By adjusting the upper cutoff, the calculated $g(0, t)$ assumes the value 1 for $t=0$. In Fig. 6 we plot the experimental values of $\left\langle h^{2}\right\rangle g(0, t)$ together with the theoretically calculated curves.

Finally we mention the initial behavior of $g(0, t)$. From expansion (A15) we deduce

$$
g(0, t)=1-\frac{t}{t_{c}} \frac{\kappa}{\ln \left(1+\kappa^{2}\right)}+\cdots .
$$

Here one sees that a finite $\kappa$ is essential for this initial behavior.

\section{PROBABILITIES ON HISTORIES}

The noise term comes into the picture when we calculate the distribution of the $h_{\mathbf{k}}(t)$. It follows from the FokkerPlanck equation, which is equivalent with the Langevin equation and reads ${ }^{23}$

$$
\frac{\partial P\left(h_{\mathbf{k}}, t\right)}{\partial t}=\omega_{k} \frac{\partial h_{\mathbf{k}} P\left(h_{\mathbf{k}}, t\right)}{\partial h_{\mathbf{k}}}+\frac{\Gamma_{\mathbf{k}}}{2} \frac{\partial^{2} P\left(h_{\mathbf{k}}, t\right)}{\partial h_{\mathbf{k}}^{2}} .
$$

It gives the evolution of the probability distribution $P\left(h_{\mathbf{k}}, t\right)$ starting from an initial distribution $P\left(h_{\mathbf{k}}, 0\right)$. The solution ${ }^{23}$ of Eq. (42) provides the conditional probability of the mode $h_{\mathbf{k}}(t)$, starting with the value $h_{\mathbf{k}}(0)$ as follows:

$$
\begin{aligned}
P\left(h_{\mathbf{k}}(0) \mid h_{\mathbf{k}}(t)\right)= & \frac{1}{\left[2 \pi\left\langle\left|h_{\mathbf{k}}\right|^{2}\right\rangle\left(1-e^{-2 \omega_{k} t}\right)\right]^{1 / 2}} \\
& \times \exp -\frac{\mid h_{\mathbf{k}}(t)-h_{\mathbf{k}}(0) e^{-\left.\omega_{k^{t}}\right|^{2}}}{2\left\langle\left|h_{\mathbf{k}}\right|^{2}\right\rangle\left(1-e^{-2 \omega_{k} t}\right)} .
\end{aligned}
$$

The expression shows that, independent of the value of $h_{\mathbf{k}}(0)$, the distribution asymptotically approaches equilibrium distribution (5).

For the measurements at different times (and possibly different positions) we need the multiple time correlation function

$$
\begin{aligned}
& G_{n}\left(h_{1}, \mathbf{x}_{1}, t_{1} ; \ldots ; h_{n}, \mathbf{x}_{n}, t_{n}\right) \\
& \quad=\left\langle\delta\left(h\left(\mathbf{x}_{1}, t_{1}\right)-h_{1}\right) \cdots \delta\left(h\left(\mathbf{x}_{n}, t_{1}\right)-h_{n}\right)\right\rangle,
\end{aligned}
$$


giving the probability of a history that the interface is at time $t_{1}$ and position $\mathbf{x}_{1}$ at a height $h_{1}$ and subsequently at time $t_{i}$ and position $\mathbf{x}_{i}$ at height $h_{i}$. In order to evaluate these correlation functions we have to translate the field $h(\mathbf{x}, t)$ into its Fourier components $h_{\mathbf{k}}$. Then we have to use the joint probability on a set of components $h_{\mathbf{k}}\left(t_{1}\right), \ldots, h_{\mathbf{k}}\left(t_{n}\right)$, which is given by the product of equilibrium probability (8) for the first event at $t_{1}$ and the conditional probabilities (43) for the successive time intervals, $t_{2}-t_{1}, \ldots, t_{n}-t_{n-1}$. The result of the integration can be derived easily from the observation that the $h\left(\mathbf{x}_{j}, t_{j}\right)$ are, as linear combinations of the basic variables $h_{\mathbf{k}}$, also Gaussian random variables. So, similar to the derivation of Eq. (12), their distribution must be of the form

$$
G_{n}\left(h_{1}, \ldots, t_{n}\right)=\left(\frac{\operatorname{det} g^{-1}}{\left(2 \pi\left\langle h^{2}\right\rangle\right)^{n}}\right)^{1 / 2} \exp \left[-\frac{1}{2} \sum_{i, j} g_{i, j}^{-1} \frac{h_{i} h_{j}}{\left\langle h^{2}\right\rangle}\right]
$$

The matrix $g_{i, j}$ is the correlation matrix

$$
g_{i, j}=g\left(\left|\mathbf{x}_{i}-\mathbf{x}_{j}\right|, t_{i}-t_{j}\right) .
$$

The proof of Eq. (45) is exactly the same as that of Eq. (12).

Equation (45) is the main result of the theory for the histories. It relates the probability of a history $h_{1}, \ldots, h_{n}$ on a sequence of snapshots to the height-height correlation function $g(x, t)$. The strong point of Eq. (45) is that the timedependent probability densities have exactly the same structure as the equal-time probabilities, when expressed in the appropriate $g_{i, j}$. Thus the whole analysis given above for the equal-time correlations can be taken over for the more general correlations. So we restrict ourselves for the timedependent histories to the aspects needing some extra attention.

The time-dependent probability density $G_{2}$ reads as Eq. (20) with $g=g(0, t)$. It can also be written as the product of the equilibrium distribution $P_{\mathrm{eq}}\left(h_{1}\right)$ and the conditional probability $G_{c}\left(h_{1}, 0,0 \mid h_{2}, 0, t\right)$ that starting at $h_{1}$ one arrives at $h_{2}$ at time $t$ later,

$$
G_{c}\left(h_{1}, 0,0 \mid h_{2}, 0, t\right)=\frac{1}{\left[2 \pi\left(1-g^{2}\right)\right]^{1 / 2}} \exp -\frac{\left[h_{2}-h_{1} g\right]^{2}}{2\left[1-g^{2}\right]} .
$$

This expression cannot be seen as the "propagator" for the probability, like Eq. (43) is for the Fourier components $h_{\mathbf{k}}$. While the probabilities for modes $\mathbf{k}$ evolve as a Markov process, the distribution for $h(\mathbf{0}, t)$ does have a memory effect. Only if $g(0, t)$ were a pure exponential the spatial process would be Markovian too. ${ }^{23}$ Expression (38) shows that $g(0, t)$ it is not a pure exponential but a superposition of exponentials. For longer times it starts to decay as an exponential when the slowest mode begins to dominate, as can be seen in Fig. 6.

\section{AVERAGE NUMBERS OF HILLS AND VALLEYS}

Consider now a sequence of snapshots, taken with time intervals $\Delta t$. We are again interested in the probabilities on the duration of hills and valleys with respect to a level $h$. To stress the analogy between space and time we use the same

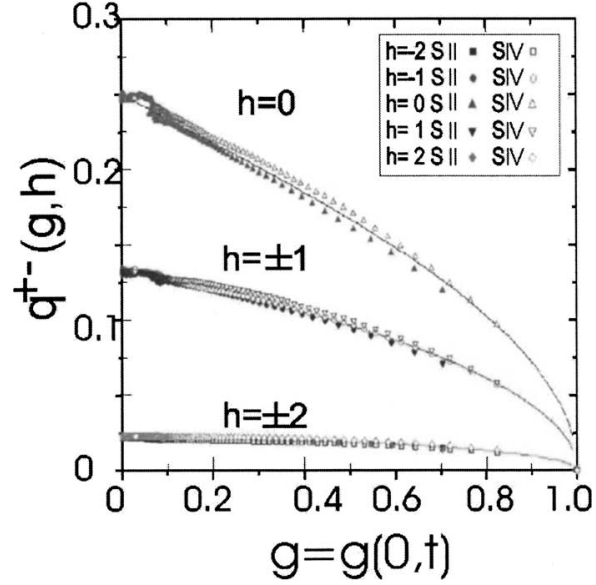

FIG. 7. The function $q^{+-}$as a function of $t$ represented by $g=g(0, t)$, for $h=-1$ (filled squares), $h=0$ (circles), and $h=1$ (semifilled pentagons). The drawn lines are calculated values and the points are the measured values.

notation $p_{n}^{ \pm}(h)$ for the probabilities to find a stretch of exactly $n$ consecutive values of the interface height above/ below the level $h$, where $n$ now is an index in the time direction. They are given by the same integrals as Eqs. (22) and (23) with $g_{i, j}$ the temporal correlation matrix. The mean values are called the residence time $\tau^{+}(h)\left[\right.$ for $\left.p_{n}^{+}(h)\right]$ and the waiting time $\tau^{-}(h)$ [for $\left.p_{n}^{-}(h)\right]$.

To check whether the experimental values follow these theoretical predictions, we first checked that the ratio $\tau^{+}(h) / \tau^{-}(h)$ is the same $q^{+}(h) / q^{-}(h)$ in analogy with Eq. (27). It is valid over several orders of magnitude. Only for the very large $h$ deviations occur due to poor statistics. In Fig. 7 we now plot again the calculated values of $q^{+-}(h, g)$ as a function of the parameter $g=g(0, t)$, for a number of $h$ values. The upper curve in Fig. 7 for $h=0$ is given by Eq. (29). In this figure the experimental values are plotted as follows. We take as time interval a multiple $n$ of the smallest interval $\Delta t$ and determine for this sampling rate the $\tau$ 's. This leads to experimental values of $q^{+-}(h, g)$, which we plot in the figure at the value $g=g(n \Delta t)$. The curves for a fixed value of $h$ are statepoint independent; the figure shows that this is pretty well the case.

Finally we plot in Fig. 8 the dependence of the $\tau$ 's on $h$ for three choices of the time interval. The curves are confusing at first sight, since the values of $\tau^{ \pm}(h)$ are about the same for all three choices. So, if we multiply them with the value of the chosen time interval, in order to convert them from numbers to real times, we get substantial different times. This indicates that the residence and waiting times depend strongly on the measuring process.

The fact that the smallest chosen time interval leads to the smallest value of the residence and waiting times naturally poses the following question: What will happen in the limit of vanishingly small time interval $\Delta t$ ? Theoretically it relies on the behavior of the correlation function $g(t)$ in the limit $t \rightarrow 0$. We presented in Eq. (41) the behavior as it follows from capillary wave theory. A linear approach of $g$ to 1 implies that $\tau^{+-}(h)$ increases as the inverse power of the square root of $\Delta t$. Then, after multiplying with $\Delta t$ in order to get their values in real time, the residence and waiting times 


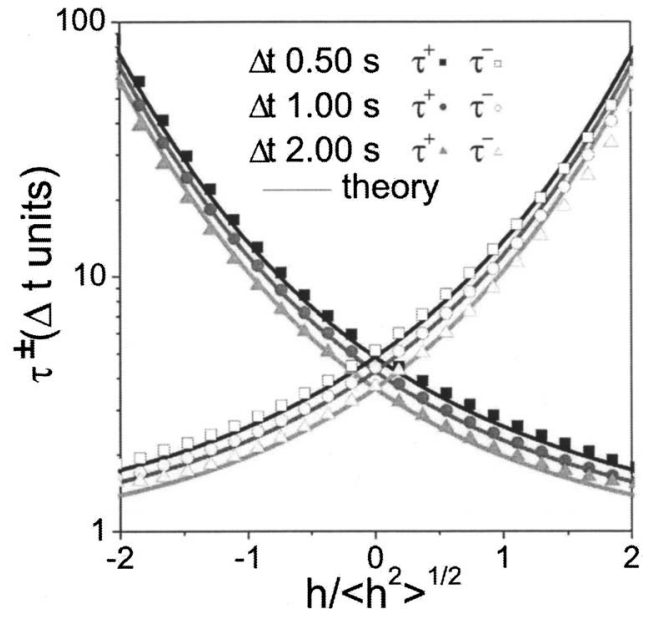

FIG. 8. Values of $\tau^{ \pm}(h)$ as a function of $h$ for three time intervals for three intervals $\Delta t, 2 \Delta t$, and $4 \Delta t$.

vanish as the square root of $\Delta t$. This problem was noticed in Ref. 16. However, the slope of the linear term in Eq. (41) depends on a molecular quantity $\kappa$, which indicates that wavelengths matter for which the mesoscopic capillary wave theory is not designed. ${ }^{5}$ One could argue that for molecular times, the cusp in $g(0, t)$ is rounded off to a parabola (since it is time reversal invariant). Then this parabola would compensate the square root in Eq. (30) and the residence and waiting times would approach a finite limit. ${ }^{16}$

Unfortunately this scenario cannot be tested experimentally, given the present limits on the sampling frequency. However, there is an interesting sampling regime beyond our data, for which the capillary wave theory still holds. In Fig. 7 the data go up to the value $g \simeq 0.8$. The typical square root decay of the curves for $q^{+-}(h, g)$ cannot be tested with our data. A microscope, which is faster by a factor 10 , could enter this regime where the typical signature of the Brownian character of the fluctuations is most significant. They give increasingly larger weight to short living hills and valleys, which force the mean values to shrink in a specific way predicted by the presented theory.

The same issue presents itself in the analysis of the data for a single time as a function of the sampling distance, but in a less severe way. In the Appendix it is shown that the height-height correlation as a function of the distance is a parabola for short distances. Thus a finite value of the sequence length and the recurrence length would follow in the limit of continuous sampling. However, again we do not reach the regime and the theoretical limiting values are strongly dependent on the cutoff $\kappa$, where the capillary wave theory breaks down.

\section{DEPENDENCE OF $p_{n}^{ \pm}(h)$ ON $n$ AND $h$}

We plot in Fig. 9 the experimental curves for $p_{n}^{+}(h)$ for a large number of $n$ for statepoint IV. A noteworthy point is that only for rather large values of $n$ the decay with $n$ (time) becomes exponential. To calculate the persistence exponent governing the decay is the challenging problem alluded to in Sec. I.

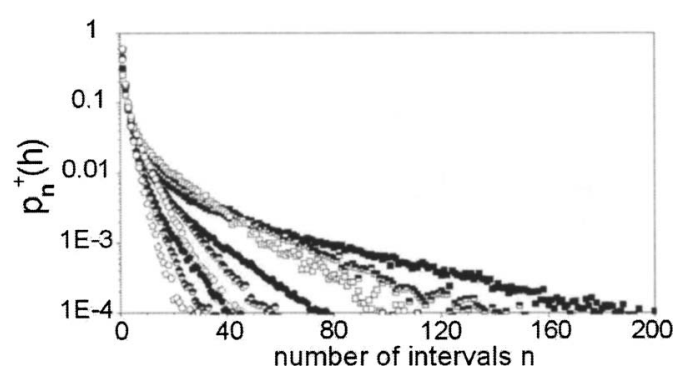

FIG. 9. The temporal $p_{n}^{+}(h)$ for statepoint IV, for $h=-1$ (squares), $h=0$ (circles), and $h=1$ (pentagons). The fillings correspond to time intervals $\Delta t=0.5 \mathrm{~s}$ (open), $2 \Delta t$ (semifilled), and $4 \Delta t$ (filled).

The scatter in the data is modest, even for large $n$ corresponding to large times $t$. Thus the experiment provides a host of detailed information on the statistics of the fluctuations in a wide time range.

Another way of plotting the data is to select one value of $n$ and plot $p_{n}^{ \pm}(h)$ as a function of $h$. Figure 10 shows the experimental data for statepoint IV for $p_{n}^{+}(h)$.

This way of presenting the data facilitates the comparison with the theory. The drawn lines are the theoretical values as given by Eq. (21). We reiterate that the input in the calculations is a set of $n+2$ experimental values of $g(0, t)$. The agreement between theory and experiment is remarkable for these detailed data. Statepoint II gives similar results with a slight asymmetry between up and down, of the same type as deviations in the spatial correlation functions, shown in Fig. 5.

The data for $p_{n}^{-}(h)$ have been independently collected. Symmetry (25) is very well obeyed, such that there is no point in showing these data separately.

\section{DISCUSSION}

The above given analysis of the statistics of interface fluctuations naturally falls into two parts, in which the height-height correlation function $g(x, t)$ plays a pivotal role. The first part concerns the connection between $g(x, t)$ and the state parameters such as $\Delta \rho, \eta$, and $\gamma$. The second part is the determination of the multiple correlation functions $G\left(h_{1}, \ldots, t_{n}\right)$ from $g(x, t)$ through Eq. (45). In the first part we have used the data for $g(x, t)$ to determine the state parameters. Even though the derivation of the structure of

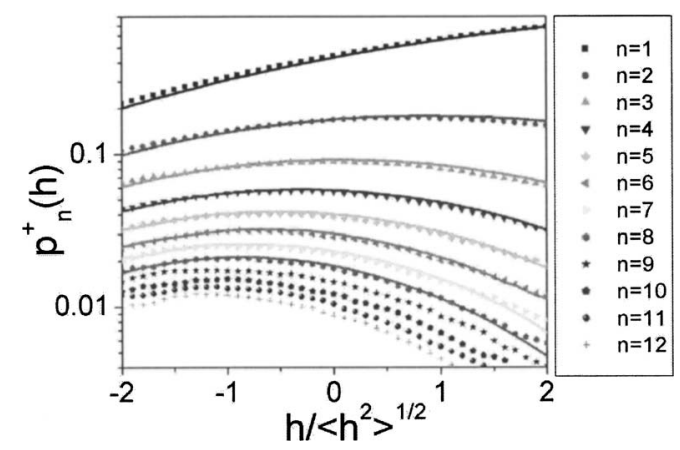

FIG. 10. The temporal $p_{n}^{+}(h)$ as a function of $h$ for a number of $n$ for statepoint IV. The drawn lines are the theoretical values. 
$g(x, t)$ in space and in time is quite different, the behavior is remarkably similar if the space and time variables are properly scaled [see Eqs. (10) and (40)].

The second part has been our main concern. We used the measured $g(x, t)$ as input, providing all the necessary information on the statistics of the snapshots. The advantage of splitting the problem into these two parts is that the second part is not confounded by errors in the first. The only assumption in the theory is the use of the Langevin equation for the effect of the thermal (white) noise. The best justification for this procedure is a posteriori through its consequences. In view of the successful agreement with the experimental results, the assumption appears to be very well fulfilled.

Experimentally the capillary waves are disentangled from the structure of the intrinsic interface. Most amazing is that detailed correlations in capillary waves can be determined with high accuracy. The statistics of the temporal dependence is generally better than that of the spatial behavior. We have chosen only a limited set of obtainable correlation functions in order to compare them with the theoretical calculations. Experimentally it is easy to collect data for practically any interesting $n$. In Figs. 5 and 9 we show the distribution $p_{n}^{+}(h)$ as a function of $n$ for the values $h=-1,0$, and 1.

There is a simplifying aspect in the fact that experimentally only sequences of finite time intervals can be measured. So one does not know what the interface does in between two snapshots. But this is precisely the reason why it suffices to calculate the correlation functions defined in Sec. IV. Here also one does not specify the evolution in between two snapshots. For instance, the key quantity $q^{+-}(h, g)$ for the residence and waiting times involves the crossing of the level $h$ by the interface. But it does not say that it may cross it only once! Any odd number of crossings is possible. In Fig. 7, where we compare $q^{+-}(h, g)$ with experiment, large time intervals feature (corresponding to small $g$ ) and in these large time intervals crossings are frequently taking place. Also the hills and valleys of length $n$, for which the distribution is given in Figs. 5 and 10, may be interrupted by opposite values in between snapshots. The charm of the comparison is that both theory and experiment allow these possibilities.

Of course, even though overall the comparison between theory and experiment is very good, upon close inspection there are always slight differences, some of which seem to be systematic. E.g., as we noted in the discussion of Fig. 2, fitting the distribution of heights gives a 1\%-3\% smaller value for $\left\langle h^{2}\right\rangle$ than when this average is determined directly from the data. Likewise, in Fig. 5 there appear to be small systematic deviations between theory and experiments for large heights, and in Fig. 6 for early times for the lower curve corresponding to statepoint IV. We are at this point not sure about the origin of these discrepancies, but wonder whether these could be due to coupling to decomposition modes associated with the fact that we have a binary liquid. ${ }^{24}$ Further study will be needed to settle these issues.

In this paper we have restricted ourselves to sequences of height measurements at the same time or at the same position. General result (45) shows that one could equally well correlate snapshots at combinations of times and positions and do a similar statistical analysis. The only point that matters is the height-height correlation $g_{i, j}$ between the events $i$ and $j$. Also one does not need to worry in how much the measurements refer to a single point or to an area of finite size. These more collective variables are also linear combinations of the basic variables $h_{\mathbf{k}}$ and therefore also Gaussian randomly distributed. Then taking the measured correlations between the more general variables as input leads to exactly the same analysis as given here for point variables.

Theoretically remains the problem of calculating the asymptotic decay of the probabilities $p_{n}^{ \pm}(h)$ for large $n$ from the measured height-height correlation function $g(x, t)$. The fact that the first few calculated values $(n<9)$ agree quite well with the measured data encourages to attack this (classic unsolved $^{16,17}$ ) problem in this specific context where ample experimental data are available.

\section{ACKNOWLEDGMENTS}

The authors are indebted for valuable discussions with D. G. A. L. Aarts, H. W. J. Blöte, G. T. Barkema, and members of the "Theorieclub," where this problem arose. W.v.S. and J.M.J.v.L. thank J. D. Weeks for pointing out the connection with the persistence problem. We also thank J. de Folter, C. Vonk, S. Sacanna, and B. Kuipers for help with the synthesis and characterization of the experimental system. The work of V.W.A.d.V. is part of the research program of the "Stichting voor Fundamenteel Onderzoek der Materie (FOM)," which is financially supported by the "Nederlandse Organisatie voor Wetenschappelijk Onderzoek (NWO)." Support of V.W.A.d.V. by the DFG through the SFB TR6 is acknowledged.

\section{APPENDIX: THE HEIGHT-HEIGHT CORRELATION INTEGRAL}

In this appendix we discuss some properties of the height-height correlation function $g(x, t)$. We start with the equal-time function $g(x, 0)$. Using the scaled integration variable $y=k \xi$ the integral for $g(x, 0)$ leads to

$$
g(x, 0)=\frac{2}{\log \left(1+\kappa^{2}\right)} \int_{0}^{\kappa} y d y \frac{J_{0}(x y / \xi)}{1+y^{2}}
$$

where $\kappa=k_{\max } \xi$ is the cutoff. Sending this value to $\infty$ yields the modified Bessel function $K_{0}$ as follows:

$$
\int_{0}^{\infty} y d y \frac{J_{0}(x y / \xi)}{1+y^{2}}=K_{0}(x / \xi) .
$$

For finite $\kappa$ we can make a short distance expansion, reading

$$
\begin{aligned}
\int_{0}^{\kappa} y d y \frac{J_{0}(x y / \xi)}{1+y^{2}}= & \frac{1}{2} \log \left(1+\kappa^{2}\right) \\
& +\left(\frac{x}{\xi}\right)^{2}\left(\frac{\kappa^{2}-\log \left(1+\kappa^{2}\right)}{2}\right)+\cdots .
\end{aligned}
$$

Matching the small argument expansion of $K_{0}(x / \xi)$, 


$$
K_{0}(x / \xi)=\log (x / 2 \xi)-0.57721+\cdots
$$

with Eq. (A3) gives for the point where they cross the approximate value

$$
x \simeq \frac{\xi}{\kappa} .
$$

The behavior in the time direction is remarkably similar to the spatial direction, although the integral for $g(0, t)$ looks quite different. We write

$$
g(0, t)=\frac{2}{\log \left(1+\kappa^{2}\right)} H\left(t / t_{c} ; \kappa\right),
$$

with $H(z ; \kappa)$ as the integral

$$
H(z ; \kappa)=\int_{0}^{\kappa} \frac{y d y}{1+y^{2}} \exp \left[-z\left(y+y^{-1}\right) / 2\right] .
$$

The first point is to prove relation (40), which we do by showing that

$$
\frac{d H(z, \infty)}{d z}=-K_{1}(z),
$$

and checking the asymptotic expansion of Eq. (40) for large $z$. The advantage of Eq. (A8) is that

$$
\frac{d H(z ; \infty)}{d z}=-\frac{1}{2} \int_{0}^{\infty} d y \exp \left[-z\left(y+y^{-1}\right) / 2\right]
$$

is a simpler integral than Eq. (A7). Then take $x=\ln y$ as integration variable, which turns Eq. (A9) into

$$
\frac{d H(z ; \infty)}{d z}=-\frac{1}{2} \int_{-\infty}^{\infty} d x e^{x} \exp (-z \cosh x) .
$$

Splitting the integral into pieces from $-\infty$ to 0 and from 0 to $\infty$ and changing in the first part from $x$ to $-x$ yield the relation

$$
\frac{d H(z ; \infty)}{d z}=-\int_{0}^{\infty} d x \cosh x \exp (-z \cosh x) .
$$

The integral is a representation of the function $K_{1}(z){ }^{25}$

In order to show that no constant is lost in going from Eq. (40) to Eq. (A8) one can check the asymptotic expansion of Eq. (40) for large $z$, which follows from an expansion around the slowest mode for $y=1$ as follows:

$$
y+y^{-1}=2+(y-1)^{2}+\cdots,
$$

and replacing the integral with a full Gaussian around $y=1$. Then one gets

$$
\begin{aligned}
H(z ; \infty) & \simeq \frac{e^{-z}}{2} \int_{-\infty}^{\infty} d(y-1) \exp \left[-(y-1)^{2} z / 2\right] \\
& =e^{-z\left(\frac{\pi}{2 z}\right)^{1 / 2}},
\end{aligned}
$$

which matches the asymptotic expansion of $K_{0}(z)$.

The final point is the expansion for small times $t / t_{c}$ for a finite value of $\kappa$. We expand the exponential

$$
\exp \left[-\left(y+y^{-1}\right) z / 2\right]=1-\left(y+y^{-1}\right) z / 2+\cdots
$$

and insert this expansion into integral (A7). Then we find for $H(z ; \kappa)$

$$
H(z ; \kappa)=\frac{1}{2} \ln \left(1+\kappa^{2}\right)-\frac{\kappa}{2} z+\cdots .
$$

Note that the next term in this expansion leads to a logarithmically divergent integral at the small $y$ side. Thus the next term is not of the order $z^{2}$ but of the order $z^{2} \ln z$. The finite $\kappa$ integral stays finite in contrast to $K_{0}(z)$, which diverges for $z=0$. With expansions (A4) and (A15) we find for the point where the finite- $\kappa$ curve starts to deviate from the $K_{0}(z)$ as follows:

$$
t \simeq t_{c} / \kappa
$$

One obtains the rough estimate $z \simeq 1 / \kappa$ for this matching point, by looking to the value of the exponential at the upper boundary, which is $\exp \left[-z\left(\kappa+\kappa^{-1}\right) / 2\right]$. For larger values the boundary value starts to vanish and extending the integral to infinity leads to a small error. For smaller values of this $z$, the exponent of the exponential becomes smaller than 1 at the boundary and the integrand of Eq. (A7) has not yet died out at $y=\kappa$. Then deviations from the infinite domain start to show up.

${ }^{1}$ J. D. van der Waals, Z. Phys. Chem. 13, 657 (1894).

${ }^{2}$ M. V. von Smoluchowski, Ann. Phys. (N.Y.) 25, 205 (1908).

${ }^{3}$ L. Mandelstam, Ann. Phys. (N.Y.) 41, 609 (1913).

${ }^{4}$ F. P. Buff, R. A. Lovett, and F. H. Stillinger, Phys. Rev. Lett. 15, 621 (1965).

5 J. D. Weeks, J. Chem. Phys. 67, 3106 (1977).

${ }^{6}$ C. V. Raman, Nature (London) 112, 281 (1923).

${ }^{7}$ A. Vrij, Adv. Colloid Interface Sci. 2, 39 (1968).

${ }^{8}$ A. Braslau, P. S. Pershan, G. Swislow, B. M. Ocko, and J. Als-Nielsen, Phys. Rev. A 38, 2457 (1988).

${ }^{9}$ M. K. Sanyal, S. K. Sinha, K. G. Huang, and B. M. Ocko, Phys. Rev. Lett. 66, 628 (1991).

${ }^{10}$ C. Fradin, A. Braslau, D. Luzet, D. Smilgies, M. Alba, N. Boudet, K. Mecke, and J. Daillant, Nature (London) 403, 871 (2000).

${ }^{11}$ J. H. Sikkenk, J. M. J. van Leeuwen, E. O. Vossnack, and A. F. Bakker, Physica A 146, 622 (1987).

${ }^{12}$ D. G. A. L. Aarts, M. Schmidt, and H. N. W. Lekkerkerker, Science 304, 847 (2004).

${ }^{13}$ D. Derks, D. Aarts, D. Bonn, H. N. W. Lekkerkerker, and A. Imhof, Phys. Rev. Lett. 97, 038301 (2006).

${ }^{14}$ C. P. Royall, D. G. A. L. Aarts, and H. Tanaka, Nat. Phys. 3, 636 (2007).

${ }^{15}$ D. G. A. L. Aarts and H. N. W. Lekkerkerker, J. Fluid Mech. 606, 275 (2008).

${ }^{16}$ J. Krug, H. Kallabis, N. J. Majumdar, S. J. Cornell, A. J. Bray, and C. Sire, Phys. Rev. E 56, 2702 (1997).

${ }^{17}$ S. N. Majumdar and C. Sire, Phys. Rev. Lett. 77, 1420 (1996).

${ }^{18}$ V. W. A. de Villeneuve, J. M. J. van Leeuwen, J. W. J. de Folter, D. G. A. L. Aarts, W. van Saarloos, and H. N. W. Lekkerkerker, Europhys. Lett. 81, 60004 (2008).

${ }^{19}$ B. Vincent, Colloids Surf. 50, 241 (1990).

${ }^{20}$ H. N. W. Lekkerkerker, W. C. K. Poon, P. N. Pusey, A. Stroobants, and P. B. Warren, Europhys. Lett. 20, 559 (1992).

${ }^{21}$ Although the drumhead Hamiltonian (4) is well known and studied, it is useful to remind the reader that the reality of the height function $h(r)$ implies that $h_{\mathbf{k}}=h_{-\mathbf{k}}$ so that the fluctuations at $\mathbf{k}$ and $-\mathbf{k}$ are not independent; as a result care should therefore be taken in calculating averages like in Eq. (6). We refer to R. Kayser, Phys. Rev. A 33, 1948 (1986), for a detailed discussion of these points. 
${ }^{22}$ U.-S. Jeng, L. Esibov, L. Crow, and A. Steyerl, J. Phys.: Condens. Matter 16S, 4231 (1998).

${ }^{23}$ N. G. van Kampen, Stochastic Processes in Physics and Chemistry, 3rd ed. (Elsevier, New York, 2007).
${ }^{24}$ T. Hiester, S. Dietrich, and K. Mecke, J. Chem. Phys. 125, 184701 (2006).

${ }^{25}$ M. Abramowitz and I. E. Stegun, Handbook of Mathematical Functions, 9th ed. (Dover, New York, 1972). 